\title{
Avaliação funcional das fraturas intra-articulares do calcâneo tratadas cirurgicamente ${ }^{*}$
}

\author{
Functional evaluation of surgically-treated \\ intra-articular fractures of the calcaneus
}

\begin{abstract}
Cristine Mildred de Liz Medeiros ${ }^{1}$, Juan Esteban Suarez HenaO², Caroline Rohenkohl'3 ,

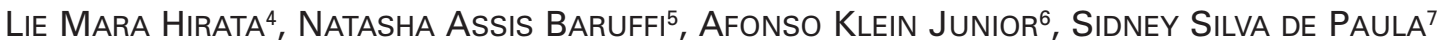

\section{RESUMO}

Objetivo: Realizar uma avaliação clínica e funcional das fraturas intra-articulares do calcâneo tratadas cirurgicamente pelo Grupo de Cirurgia do Pé e Tornozelo do Serviço de Ortopedia e Traumatologia do Hospital Universitário Cajuru no período compreendido entre janeiro de 2001 e dezem-

* Trabalho realizado pelo Grupo de Cirurgia do Pé e Tornozelo do Departamento de Ortopedia e Traumatologia do Hospital Universitário Cajuru - HUC - Curitiba (PR), Brasil.

1. Residente de Terceiro Ano de Ortopedia e Traumatologia do Hospital Universitário Cajuru - HUC - Curitiba (PR), Brasil.

2. Médico Especializando de Terceiro Ano de Ortopedia e Traumatologia do Hospital Universitário Cajuru - HUC - Curitiba (PR), Brasil.

3. Acadêmica de Medicina e Estagiária do Serviço de Ortopedia e Traumatologia do Hospital Universitário Cajuru - HUC - Curitiba (PR), Brasil.

4. Acadêmica de Medicina e Estagiária do Serviço de Ortopedia e Traumatologia do Hospital Universitário Cajuru - HUC - Curitiba (PR), Brasil.

5. Acadêmica de Medicina e Estagiária do Serviço de Ortopedia e Traumatologia do Hospital Universitário Cajuru - HUC - Curitiba (PR), Brasil.

6. Médico Assistente do Grupo de Cirurgia do Pé e Tornozelo do Hospital Universitário Cajuru - HUC - Curitiba (PR), Brasil.

7. Mestre, Chefe do Grupo de Cirurgia do Pé e Tornozelo do Hospital Universitário Cajuru - HUC - Curitiba (PR), Brasil.

Endereço para correspondência: Cristine Mildred de Liz Medeiros, Praça Osório, 225, apto. 401 - 80020-010 - Curitiba (PR), Brasil. Tel.: (41) 9193-2173, fax (41) 3271-2930.

E-mail:mimi_medeiros@hotmail.com

Recebido: 12.6.08. Aprovado: 27.11.08.

Copyright RBO2008 bro de 2006. Métodos: 107 pacientes, com 113 fraturas, foram submetidos ao tratamento conforme a classificação de Essex-Lopresti: nas fraturas tipo língua ou depressão central com cominuição intraarticular utilizaram-se redução aberta e fixação interna com placa para calcâneo $\left(\right.$ Synthes $^{\circledR}$ ) ou duplo H; nas demais fraturas tipo depressão central realizou-se tratamento minimamente invasivo e nas fraturas tipo língua, tratamento percutâneo. Foram avaliados e acompanhados clinicamente, com pontuação na tabela AOFAS, pelo mesmo grupo de cirurgiões. Resultados: Houve $\mathbf{7 3 \%}$ de resultados satisfatórios, média de $\mathbf{7 5 , 4}$ pontos, e $27 \%$ de insatisfatórios. Conclusão: A adequada redução das fraturas representa fator importante para que o paciente não tenha dor e edema residual. As fraturas do tipo depressão central foram as que resultaram em menor pontuação funcional. $O$ não restabelecimento do ângulo de Böhler também levou a importantes alterações funcionais.

Descritores - Calcâneo/lesões; Calcâneo/radiografia; Articulação talocalcânea; Fraturas ósseas/radiografia; Estudos de avaliação, estudos retrospectivos

\section{ABSTRACT}

Objective: To perform a clinical and functional evaluation of intra-articular fractures of the calcaneus treated surgically by the Group of Foot and Ankle Surgery at the Cajuru University Hospital from January 2001 to December 2006. Methods: 107 
patients, with 113 fractures, were submitted to treatment according to the Essex-Lopresti classification: in the tongue type fractures or central depression fractures with intra-articular comminution, open reduction and internal fixation with calcaneus plates (Synthes ${ }^{\circledR}$ ) or Double $H$ were used; for the other central depression type of fractures, the authors resorted to the minimally invasive treatment, and in tongue type fractures, percutaneous treatment was preferred. The patients were evaluated and clinically followed-up by the same group of surgeons, with evaluation scores according to the AOFAS table. Results: $73 \%$ of satisfactory results, with a mean of 75.4 score points, and $27 \%$ unsatisfactory results. Conclusion: Adequate reduction of the fractures is an important factor to prevent pain and residual edema. Central depression type of fractures were those that had the lowest functional score. Non-reestablishment of the Böhler angle also culminated in relevant functional alterations.

Keywords - Calcaneus/injuries; Calcaneus/radiography; Subtalar joint; Bone, fractures/radiography; Evaluation studies; Retrospective studies

\section{INTRODUÇÃO}

As fraturas do calcâneo representam $60 \%$ das fraturas do tarso e $2 \%$ do total de fraturas de todo o esqueleto. Ocorrem, em sua maioria, após trauma de grande energia, como queda de altura ou acidentes automobilísticos $^{(1)}$. Segundo Essex-Lopresti, 50\% encontramse associadas a outras fraturas, como platô tibial, quadril e coluna lombar ${ }^{(2)}$.

Segundo Sanders, em 1908, Cotton e Wilson já referiam em suas comunicações aos mal-sucedidos e incapacitantes resultados das fraturas de calcâneo, certamente conseqüência do precário conhecimento de sua fisiopatologia e dos recursos terapêuticos disponíveis ${ }^{(3)}$.

Só bem mais tarde, Essex-Lopresti desenvolveu para estas fraturas uma classificação radiológica baseada no mecanismo de trauma ${ }^{(2)}$ e Sanders outra, apoiada em estudo tomográfico ${ }^{(3)}$. Embora, segundo Contreras et al, os pioneiros a introduzir as placas e parafusos no tratamento dessas fraturas tenham sido Leriche e Ju- det, o tratamento cirúrgico utilizando a redução aberta e fixação interna só teve seu início na década de 80 , após a introdução da tomografia, que facilitou a diferenciação do tipo de fratura, desenvolvendo-se então as cirurgias realizadas através do acesso lateral, minimamente invasivo e percutâneo ${ }^{(4)}$.

A fratura do calcâneo possui implicações socioeconômicas porque a grande maioria das pessoas que apresentam esse tipo de fratura está em idade produtiva (90\%) e, em conseqüência, permanece inativa por um período de no mínimo três meses. A conseqüência da limitação física imposta como seqüela desse tipo de fratura tem repercussão econômica significativa ${ }^{(5)}$.

Este trabalho tem por objetivo realizar retrospectivamente uma avaliação clínica e funcional das fraturas intra-articulares do calcâneo tratadas cirurgicamente pelo Grupo de Cirurgia do Pé e Tornozelo do Serviço de Ortopedia e Traumatologia do Hospital Universitário Cajuru.

\section{MÉTODOS}

Entre janeiro de 2001 e dezembro de 2006, foram operados, no Hospital Universitário Cajuru de Curitiba, 200 pacientes com fratura intra-articular do calcâneo; 107 deles com 113 fraturas foram incluídos no trabalho por ter mantido o acompanhamento ambulatorial.

Para o diagnóstico das fraturas foi utilizado estudo radiográfico que incluiu as incidências: dorsoplantar, oblíquas do pé, perfil do calcâneo, com raio angulado a $10^{\circ}, 20^{\circ}, 30^{\circ}$ e $40^{\circ}$ do maléolo lateral (Broden) e axial de calcâneo (Harris). Na avaliação radiográfica inicial foi medido o ângulo de Böhler ou tuberositário, cujo valor normal encontra-se entre $25^{\circ}$ e $40^{\circ}(6)$. Esse ângulo foi mensurado no pré e pós-operatório. As demais incidências foram utilizadas para avaliar a integridade da articulação subtalar.

Apenas para alguns casos selecionados (grande cominuição articular) fizemos uso da tomografia computadorizada, realizada em dois planos: semicoronal (perpendicular à posição normal da faceta posterior do calcâneo) e plano axial (paralelo à planta do pé).

Utilizamos a classificação radiológica de Essex-Lopresti, que divide as fraturas em extra-articulares (pro- 
cesso anterior, corpo, sustentáculo do tálus, tubérculo fibular, processo lateral, tuberosidade e tubérculo medial) e intra-articulares, que são subdivididas em: tipo língua, depressão central e cominutivas. A fratura tipo língua apresenta a linha de fratura ao longo do eixo axial, com uma linha secundária a partir do ângulo de Gissane, na direção plantar medial para dorsolateral, destacando um fragmento lateral póstero-superior. $\mathrm{Na}$ depressão central a linha de fratura secundária avança através do ângulo de Gissane, contorna a face posterior da faceta posterior, formando um fragmento da tuberosidade com a maior parte da faceta articular posterior do calcâneo ${ }^{(2)}$.

Nos pacientes em que foi realizado estudo tomográfico, utilizamos a classificação de Sanders, como descrito por Rockwood et al, de acordo com as imagens coronais da faceta posterior: tipo I: até quatro fragmentos, sem desvio; tipo II: dois fragmentos intra-articulares; tipo III: três fragmentos; e as do tipo IV: quatro fragmentos ${ }^{(6)}$.

Foram obtidos dados clínicos pré-operatórios considerados como fatores de risco na evolução, tais como: diabetes, tabagismo, alcoolismo e hipertensão ${ }^{(7)}$.

Mensuramos o tempo decorrido entre o dia do trauma e o dia da realização do procedimento cirúrgico.

Como proposto por Pillai et al, em todos os pacientes tratados com redução aberta e fixação interna foram utilizados decúbito lateral, torniquete e acesso lateral de Selingson ${ }^{(8)}$. Esse acesso compreende incisão em "L", imediatamente plantar ao trajeto dos tendões fibulares, estendendo-se desde a face posterior do maléolo lateral até a região da articulação calcâneo-cubóidea. Os tendões fibulares e o nervo sural são afastados e incisa-se o ligamento calcâneo-fibular. Reflete-se a origem do extensor curto dos dedos distalmente para expor a articulação calcâneo-cubóidea. A seguir fazse a redução da fratura e fixação provisória com fios de Kirschner e após, fixação com placa duplo H (figura 1) ou placa para calcâneo $\left(\right.$ Synthes $\left.^{\circledR}\right)$. Realiza-se limpeza da ferida com soro fisiológico a $0,9 \%$ e é deixado dreno suctor. Aproxima-se subcutâneo com Poliglactina $910\left(\right.$ vicryl $\left.^{\circledR}\right)$ e a pele é fechada com mononylon ${ }^{(9)}$.

Nos casos em que foi realizado procedimento minimamente invasivo (fraturas sem cominuição articular),

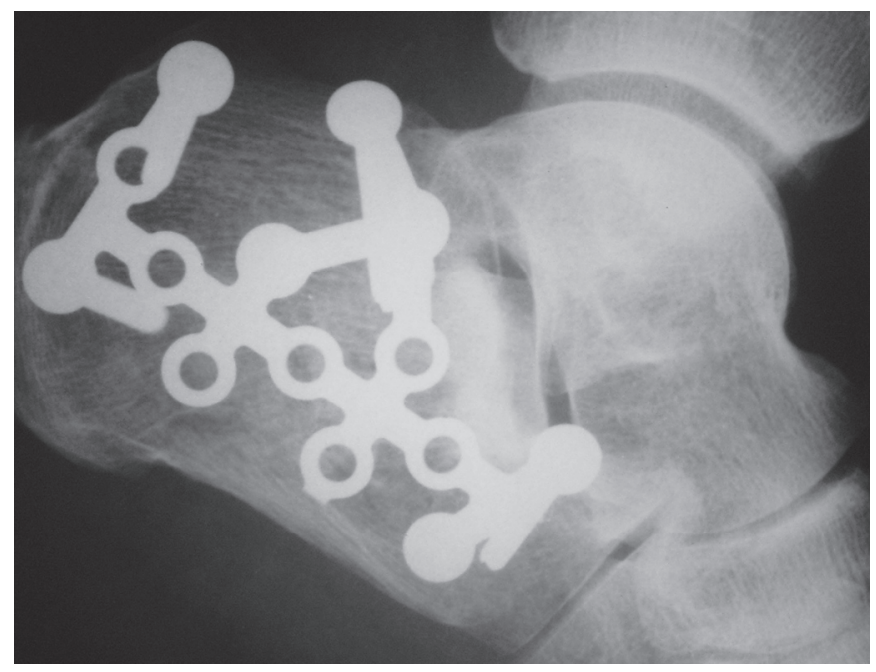

Figura 1 - Redução aberta e fixação interna com placa de calcâneo

a incisão reduz-se à pequena abertura de $2 \mathrm{~cm}$ na região subtalar lateral, com dissecção até a superfície articular. Houve casos tratados percutaneamente pela técnica de Tornetta ${ }^{(10)}$ (figura 2).

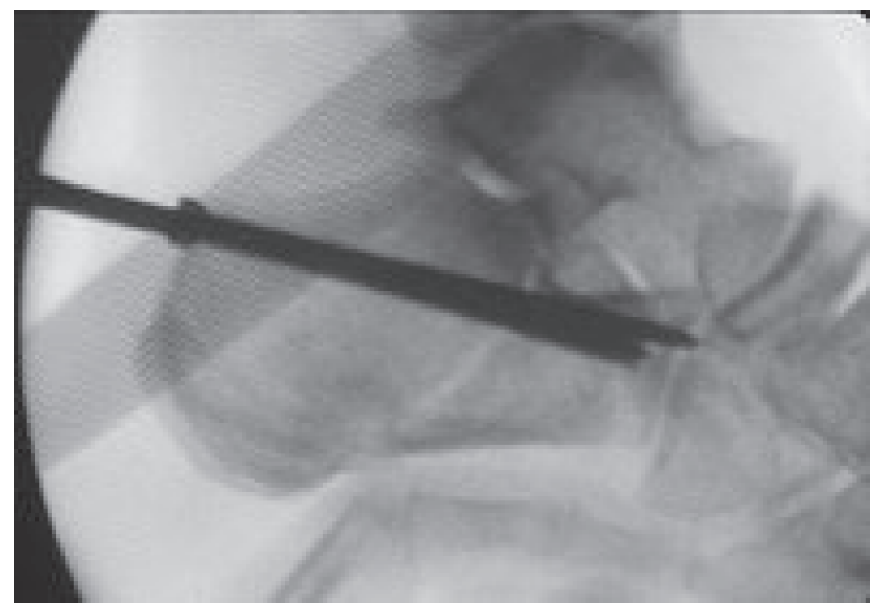

Figura 2 - Tratamento minimamente invasivo com parafuso percutâneo

Todos os pacientes receberam antibiótico - cefazolina - na dosagem de $1 \mathrm{~g}$, por via endovenosa a cada $8 \mathrm{~h}$, como antibioticoprofilaxia durante $48 \mathrm{~h}$. Nos calcâneos em que se utilizou técnica aberta com placa duplo H ou placa para calcâneo $\left(\right.$ Synthes $\left.^{\circledR}\right)$, deixou-se dreno suctor durante $24 \mathrm{~h}$. Receberam alta hospitalar logo após a suspensão da antibioticoterapia, manten- 
do-se a tala gessada suropodálica e o membro inferior elevado. Ainda no pós-operatório imediato era realizado acompanhamento da evolução da ferida cirúrgica. A primeira avaliação ambulatorial ocorreu com 15 dias de pós-operatório, quando eram retirados os pontos, analisada a ferida quanto a infecção, deiscência, necrose de pele, cicatrização, sendo, também, realizadas radiografias nos planos axial e perfil, para verificar se estava mantida a redução da fratura e avaliar o ganho de amplitude do movimento. A seguir, eram feitas novas consultas com quatro e oito semanas. A partir de dois meses, quando normalmente encontravamse sinais de consolidação, iniciou-se a fisioterapia com apoio progressivo e, aos três meses, sustentação plena do peso e treino de marcha. Nova avaliação era realizada com seis meses.

Após o tratamento cirúrgico os pacientes foram acompanhados ambulatorialmente e submetidos a uma avaliação clínica com pontuação pela escala de avaliação da American Orthopaedic Foot and Ankle Society (AOFAS), proposta por Kitaoka et al para o retropé(11). A avaliação clínica foi feita com 15 dias, um mês, dois meses e seis meses pós-operatórios.

\section{RESULTADOS}

Apenas 27\% dos pacientes (29 calcâneos) obtiveram pontuação menor que 69 pontos segundo a tabela AOFAS e $73 \%$ (78 pacientes) ficaram com pontuação maior do que 70 , sendo que seis obtiveram 100 pontos (gráfico 1). Obtivemos como média 75,4 pontos, o que é considerado como satisfatório por Thordarson et $a l^{(12)}$.

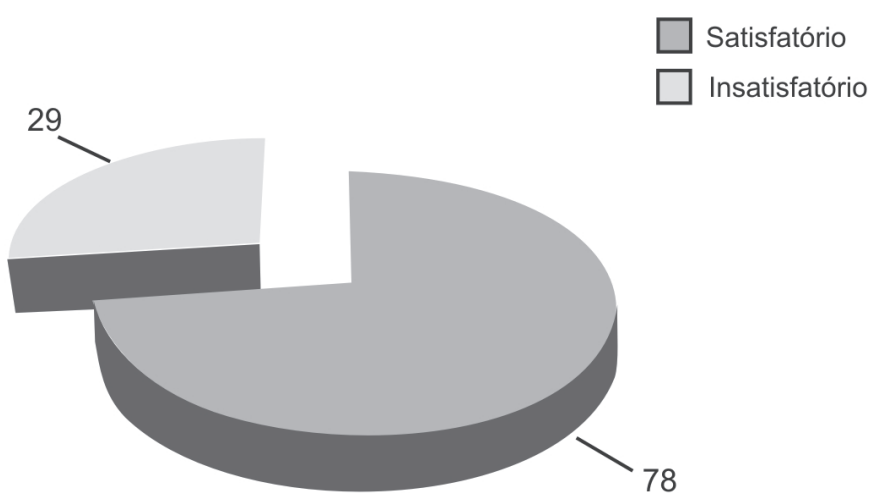

Gráfico 1 - Pontuação tabela AOFAS

Fonte: Pontuação de acordo com a escala da American Orthopedic Foot and Ankle Society
A análise foi realizada em 107 pacientes, com 113 fraturas intra-articulares do calcâneo, dentre eles 11 do sexo feminino e 96 do masculino. Em relação à idade, quatro eram menores do que 20 anos, 42 tinham entre 20 e 40 anos e 61 encontravam-se na faixa etária acima de 41 (gráfico 2).

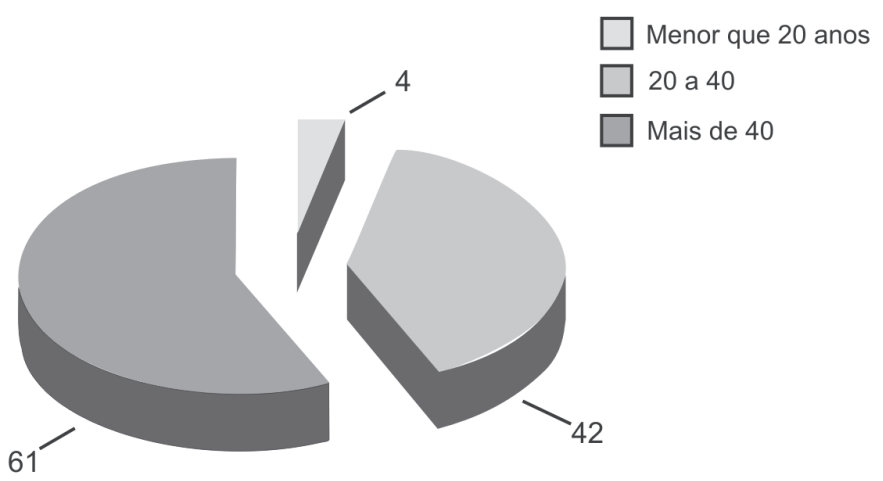

Gráfico 2 - Idade dos pacientes com fratura do calcâneo Fonte: Dados dos Prontuários dos Pacientes do Hospital Universitário Cajuru

Com relação ao mecanismo de trauma: 99 ocorreram após queda de altura, quatro por acidentes automobilísticos e quatro após trauma direto no pé. Associadas às fraturas do calcâneo, foram encontrados três casos com fratura em platô tibial, três em coluna, dois em cotovelo, dois com fraturas associadas no mesmo pé (metatarsos), dois com fratura na mão e punho, um em pilão tibial, um no fêmur e um com fratura da patela. Quanto à exposição, que, segundo Talarico et al, representa um fator de risco de partes moles, seis calcâneos sofreram fraturas expostas e 101 apresentaramse fechadas ${ }^{(13)}$. De acordo com a classificação de Essex-Lopresti, os tipos de fratura intra-articular encontrados foram: $42 \%$ cominutas, $42 \%$ depressão central e $16 \%$ tipo língua ${ }^{(2)}$ (gráfico 3 ).

Em relação ao tempo decorrido entre o dia do trauma e o momento da realização do procedimento cirúrgico: $21 \%$ foram operados no mesmo dia, $62 \%$ na mesma semana e $17 \%$ tiveram seu tratamento prorrogado para a segunda semana após o trauma.

Entre os fatores de risco para complicações pós-operatórias encontramos 20 pacientes tabagistas, um portador de diabetes mellitus, dois hipertensos e um etilista. 


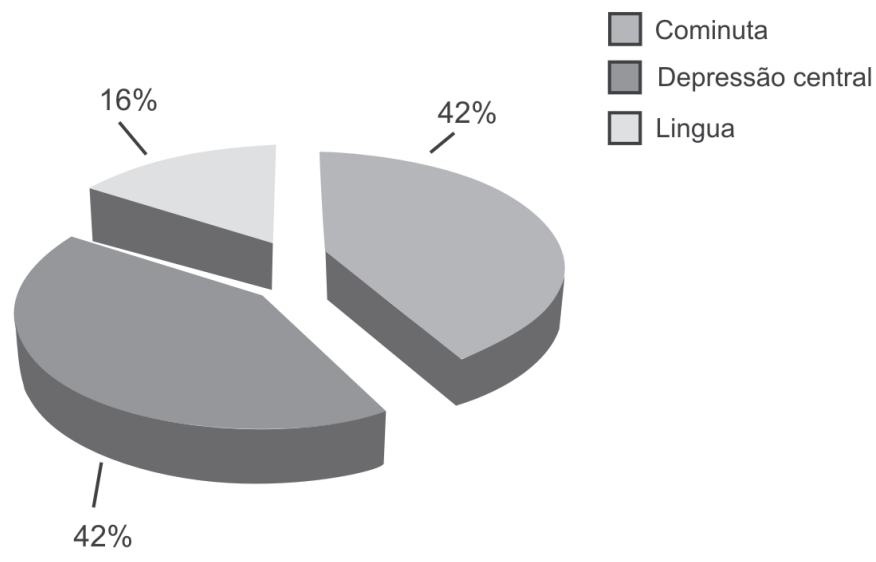

Gráfico 3 - Tipos de fratura

Fonte: Dados dos Prontuários dos Pacientes do Hospital Universitário Cajuru

Em $83 \%$ das fraturas foi empregada a técnica aberta com placa duplo H ou placa do calcâneo $\left(\right.$ Synthes $\left.^{\circledR}\right)$, em $14 \%$ a técnica minimamente invasiva e fixação com parafusos e em $3 \%$ foram utilizados fios de Kirschner, por se tratar de fraturas expostas.

Em relação às complicações precoces de partes moles, cinco pacientes apresentaram infecção no local da cirurgia, sendo que três deles eram tabagistas, nenhuma fratura fora exposta e nenhum dos pacientes era diabético. Destes cinco que infeccionaram, dois tinham sido operados no mesmo dia, dois dentro de três dias e um teve seu tratamento prorrogado para sete dias. Apenas um apresentou deiscência de sutura e este não apresentava qualquer fator de risco prévio.

Quanto às complicações tardias, dois evoluíram para artrodese decorrente de artrose e dor, sendo um deles tabagista, e três apresentaram edema residual, dos quais um deles diabético.

Após o tratamento cirúrgico, 69 dos pacientes mantiveram ângulo de Böhler dentro do valor normal, 38 com redução do ângulo e seis com ângulo maior que $40^{\circ}$ (gráfico 4). Dentre os que apresentaram redução do ângulo de Böhler, $61 \%$ eram do tipo depressão central, 23\% cominutivas e 16\% tipo língua. Nas fraturas cujo ângulo de Böhler sofreu aumento, $50 \%$ delas eram tipo depressão central e 50\% tipo língua. Correlacionando à pontuação AOFAS, $32 \%$ dos casos com diminuição do ângulo classificaram-se como insatisfatórios. E todas as que sofreram aumento do ângulo ficaram com pontuação menor que 70 na tabela AOFAS.

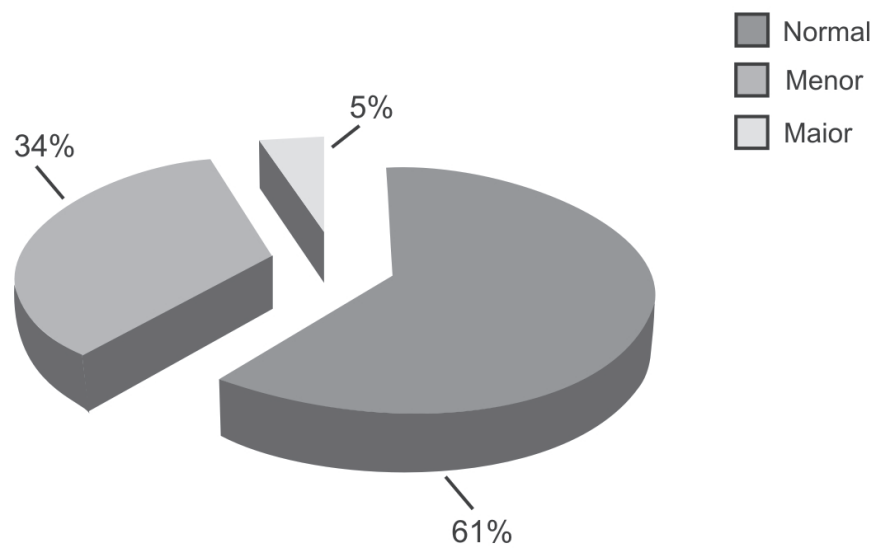

Gráfico 4 - Angulo de Böhler

Fonte: Dados dos Prontuários dos Pacientes do Hospital Universitário Cajuru

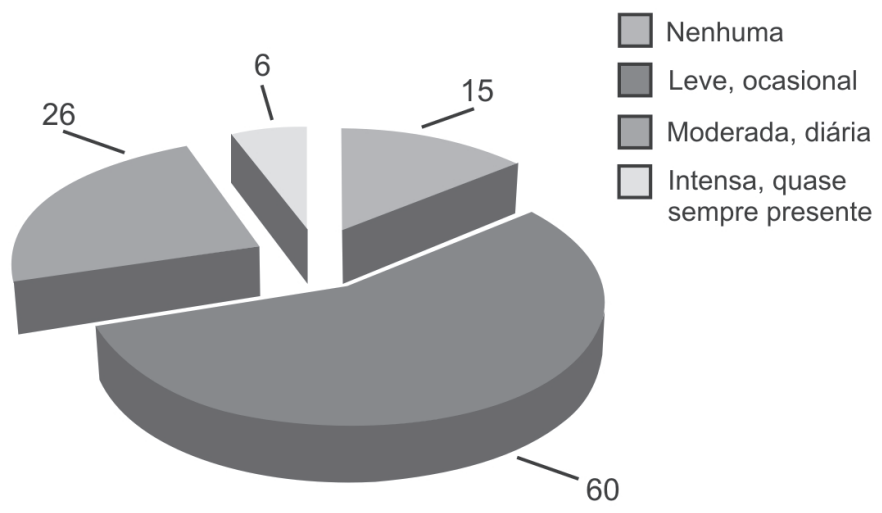

Gráfico 5 - Dor residual pós-tratamento cirúrgico

Fonte: Questionário aplicado aos pacientes do estudo em questão

Clinicamente, 15 pacientes não apresentaram qualquer dor como seqüela, 60 mantiveram dor leve, 26 dor moderada, diariamente, e seis permaneceram com dor intensa (gráfico 5). Destes últimos, duas fraturas eram tipo depressão central, duas tipo língua e duas cominutivas.

Quanto à limitação para atividades de suporte dos pacientes, oito deles apresentaram limitação importante com uso de muletas, andador, cadeira com rodas ou órtese, 17 mantiveram limitação de suas atividades, utilizando bengalas, 50 apresentaram leve limitação recreacional e 32 não apresentaram qualquer limitação.

A análise da superfície de marcha mostra que 36 pacientes não apresentaram dificuldade nas diferentes superfícies, 59 citaram leve dificuldade em terrenos irregulares, escadas, ladeiras e inclinações e 12 manti- 
veram intensa dificuldade para marcha nos terrenos irregulares.

Quanto à marcha, 45 pacientes permaneceram com capacidade de deambular mais que seis quadras; 43 andam entre quatro e seis quadras, 14 conseguem manter deambulação entre uma e três quadras e apenas cinco pacientes não andam por mais do que uma quadra.

Ao analisar a mobilidade sagital, flexão e extensão do pé, 88 pacientes mantiveram mobilidade normal ou restrição mínima de $30^{\circ}, 16$ com restrição moderada de $15^{\circ}$ a $29^{\circ}$ e três permaneceram com restrição intensa menor do que $15^{\circ}$.

A mobilidade do retropé (inversão-eversão) permaneceu normal ou com restrição leve em 41 pacientes (75-100\% de amplitude de movimento), em 44 com restrição moderada (25-74\%) e em 22 com grande restrição (menor do que $25 \%$ ).

Quanto ao alinhamento, nenhum paciente teve como seqüela o mau-alinhamento ou pé sintomático não plantígrado, 19 apresentaram pé indolor, plantígrado, com leve desalinhamento e 88 permaneceram com pé plantígrado com tornozelo e retropé alinhados.

\section{DISCUSSÃO}

Grala et al relataram o acompanhamento de 23 fraturas tratadas cirurgicamente com placas de reconstrução, descrevendo seus resultados anatômicos e funcionais em três anos de seguimento ${ }^{(14)}$. Utilizaram como meio de avaliação a superfície articular posterior e o ângulo de Böhler. Concluíram que a terapêutica adotada depende de cuidadoso planejamento pré-operatório, baseado na avaliação do tipo de fratura e condições do paciente. Destacam a necessidade de experiência do cirurgião e afirmam que uma boa ou excelente redução evita problemas ao longo do seguimento funcional do paciente. Em nossa avaliação destacamos a necessidade de manter o ângulo de Böhler dentro dos valores normais para que o paciente apresente adequada reabilitação. $\mathrm{Na}$ avaliação funcional e radiográfica após o tratamento cirúrgico, na grande maioria, $61 \%$, o ângulo de Böhler conservou-se dentro dos valores normais.

Segundo Rodriguez et al, a maioria dos pacientes que sofre este tipo de fratura apresenta traumas asso- ciados, porque o mecanismo da lesão normalmente é a queda de grandes alturas ${ }^{(1)}$. Também afirmam que as fraturas de calcâneo, devido a sua grande complexidade, devem ser tratadas por ortopedistas experientes ${ }^{(1)}$. Em nossa avaliação dos 107 pacientes, 12 apresentaram algum tipo de trauma associado.

Netzahualcóyotl Blass et al avaliaram os resultados clínico e radiológico pós-tratamento cirúrgico das fraturas do calcâneo e afirmaram ser necessário utilizar placas especiais para obter bons resultados funcionais nas fraturas intra-articulares ${ }^{(5)}$. No Hospital Cajuru utilizamos o tratamento conforme a classificação de Essex-Lopresti: nas fraturas cominutivas e nas do tipo depressão central com cominuição articular, damos preferência às placas para calcâneo (Synthes ${ }^{\circledR}$ ) e duplo $\mathrm{H}$; nas sem cominuição articular, optamos pelo acesso minimamente invasivo; e nas do tipo língua, ao tratamento percutâneo (2).

O tratamento cirúrgico percutâneo é destacado por Schepers et al após avaliação retrospectiva de 61 calcâneos em 50 pacientes ${ }^{(15)}$. Eles identificaram que as complicações pós-operatórias, como infecção e artrose secundária, foram similares às do tratamento cirúrgico aberto, porém houve excelentes resultados na preservação da mobilidade subtalar ${ }^{(15)}$.

$\mathrm{Wu}$ et al avaliaram as fraturas intra-articulares tratadas cirurgicamente com redução aberta e fixação interna $^{(16)}$. Dos casos, $80 \%$ obtiveram redução anatômica. Os autores finalizaram seu trabalho recomendando a utilização da redução aberta e fixação com placas para as lesões tipo II e III da classificação de Sanders.

Ao avaliarem as fraturas do calcâneo em idosos, Herscovici et al afirmam ser a redução aberta com fixação interna uma boa opção para o tratamento ${ }^{(17)}$. Porém, em pacientes com osteopenia, que já não se encontram em condições para deambular ou com capacidade apenas para movimentar-se dentro de casa ou ainda sem condições cirúrgicas, deve ser mantido o tratamento conservador. Não avaliamos esta particularidade porque a maioria de nossos pacientes era do sexo masculino, em idade produtiva.

Nos pacientes com fraturas expostas utilizamos primariamente o debridamento e irrigações associados à fixação com fios de Kirschner. Aldridge et al, no ano 
de 2004, ressaltaram a possibilidade de estabilização definitiva somente após boas condições de partes moles, iniciando o tratamento com irrigações e debridamentos $^{(18)}$. Quanto à utilização de fixação externa nessas fraturas, em 2004, Talarico et al ${ }^{(13)}$ relataram a possibilidade de tratamento por seis semanas em média, obtendo como complicações apenas infecção superficial.

Contreras et al realizaram uma avaliação biomecânica das fraturas intra-articulares de calcâneo. Esse estudo mostrou diferenças significantes entre o retropé e o antepé em relação à área de contato, pressão e força de reação do solo. Encontraram maiores valores no retropé fraturado. Afirmaram que, quanto melhor a redução do ângulo de Gissane, melhor a impulsão e que, quanto melhor o resultado clínico (pontuação AOFAS), melhor o apoio do retropé (primeiro pico de força $)^{(4)}$.

Quanto aos tipos de fraturas intra-articulares do calcâneo, identificamos em maior número as dos tipos cominutivo e depressão central, cada um com $42 \%$ dos casos sendo apenas 16\% tipo língua. Moraes Filho et al relataram 59\% de fraturas tipo depressão articular e $41 \%$ tipo língua ${ }^{(19)}$.

Para o tratamento cirúrgico aberto, existe o consenso de aguardar certo tempo entre o trauma e a operação, para que haja diminuição do edema e para a prevenção da formação de flictenas. Sanders et al descreveram o sinal do enrugamento da pele, significando que houve diminuição do edema ${ }^{(3)}$. Em nossa casuística, a grande maioria dos pacientes foi operada na mesma semana em que ocorreu a fratura; apenas $17 \%$ foram prorrogados para a segunda semana por diversas causas, como politraumatismo, espera de melhora de partes moles e de melhora clínica do paciente.

Como sequiela, a dor residual já descrita por Sanders em grau leve foi observada em 60 dos nossos pacientes; apenas uma minoria de 15 pessoas não relatou quadro álgico ${ }^{(3)}$. E entre os pacientes classificados com dor intensa não houve diferença quanto ao tipo de fratura.

Em relação à limitação às atividades e uso de suportes, já que a fratura do calcâneo gera limitações físicas importantes ${ }^{(14)}$, apenas oito de nossos pacientes man- tiveram grave limitação, com uso de muletas ou andador, 50 deles permaneceram com limitação recreacional e 32 evoluíram muito bem, sem necessidade de qualquer limitação. Dentre os oito que evoluíram com seqüelas, um permaneceu com ângulo de Böhler inferior ao desejado, dois com esse ângulo em valores superiores ao limite aceitável, um paciente apresentava fratura bilateral e um evoluiu para artrodese subtalar.

Quanto à mobilidade no flanco sagital, 88 mantiveram flexo-extensão normal e somente três deles permaneceram com restrição intensa. Já em relação à mobilidade do retropé, 44 pacientes permaneceram com moderada restrição à inversão-eversão e 41 mantiveram-se normais. Köberle et al relataram 21,73\% de limitação da mobilidade do tornozelo, com 43,47\% de bloqueio da articulação subtalar ${ }^{(20)}$. Santin et al ${ }^{(21)}$ descreveram algum grau de restrição na mobilidade do retropé em 67,9\% e Murachovski et al ${ }^{(22)}$, em 94,6\%.

$\mathrm{O}$ edema residual e o mau alinhamento do pé e retropé podem trazer limitações para marcha e para utilização de calçados. Murachovsky et al ${ }^{(22)}$ apresentaram $2,7 \%$ de mau alinhamento e edema crônico. Oitenta e oito pessoas ficaram com seus pés plantígrados e alinhados, 19 apresentaram leve desalinhamento indolor e nenhum teve como seqüela o mau alinhamento.

Pela pontuação funcional da escala $\mathrm{AOFAS}^{(11)}$ entre os pacientes, 78 foram classificados satisfatoriamente e 29 obtiveram pontuação menor do que 69. Dentre os que receberam pontuação insatisfatória, $77 \%$ eram do tipo depressão central, 13\% cominutivas e $9 \%$ tipo língua. Os pacientes do Hospital Universitário Cajuru ficaram com média de 75,4 pontos na escala AOFAS.

\section{CONCLUSÕES}

A adequada redução das fraturas, através da congruência articular, representa fator importante para que o paciente permaneça com boa evolução clínica e sem complicações como dor e edema residual. As fraturas do tipo depressão central foram as que resultaram em menor pontuação funcional.

O não restabelecimento do ângulo de Böhler leva a significativas alterações funcionais. Dentre os pacientes que apresentaram importantes limitações às ativi- 
dades de suporte, $40 \%$ deles encontravam-se com este referencial alterado.

O tabagismo parece ser fator de risco para complicações pós-operatórias de partes moles, pois $60 \%$ dos que infeccionaram (três de cinco) eram tabagistas e dos dois que evoluíram para artrodese, um referia ser fumante.

O tratamento utilizado no Hospital Universitário Cajuru conforme a classificação de Essex-Lopresti obteve média de 75,4 pontos pela escala de avaliação do retropé.

\section{REFERÊNCIAS}

1. Rodríguez SR, Garduño RB, Raygoza CO. Surgical treatment of calcaneal fractures with a special titanium AO plate. Acta Ortop Mex. 2004;18(Supl 1):S34-S38.

2. Essex-Lopresti P. The mechanism, reduction technique, and results in fractures of the os calcis. Br J Surg. 1952;39(157): 395-419.

3. Sanders R. Displaced intra-articular fractures of the calcaneus. J Bone Joint Surg Am. 2000;82(2):225-50. Review.

4. Contreras MEK, Muniz MAS, Souza JB, Avila AOV, Borges Júnior NG, Barbosa DRF, et al. Avaliação biomecânica das fraturas intra-articulares do calcâneo e sua correlação clínica radiográfica. Acta Ortop Bras. 2004;12(2):105-12.

5. Netzahualcóyotl Blass JPF, Gutiérrez MI, Makkozzay PTH. Resultado clínico-radiológico del tratamiento quirúrgico de las fracturas intra-articulares del calcáneo. Acta Ortop Mex. 2004; 18(1):21-4.

6. Rockwood CA, Green DP, Bucholz RW, Heckman JD, editors. Rockwood and Green's fractures in adults. 6th ed. Philadelphia: Lippincott Williams \& Wilkins; 2006.

7. Howard JL, Buckley R, McCormack R, Pate G, Leighton R, Petrie D, Galpin R. Complications following management of displaced intra-articular calcaneal fractures: a prospective randomized trial comparing open reduction internal fixation with nonoperative management. J Orthop Trauma. 2003;17(4): 241-9.

8. Pillai A, Basappa P, Ehrendorfer S. Modified Essex-Lopresti/ Westheus reduction for displaced intra-articular fractures of the calcaneus. Description of surgical technique and early outcomes. Act Orthop Belg. 2007;73(1):83-7.
9. Canale ST, Campbell WC. Campbell's operative orthopaedics. 10th ed. St Louis: Mosby; 2003.

10. Tornetta P 3rd. The Essex-Lopresti reduction for calcaneal fractures revisited. J Orthop Trauma. 1998;12(7):469-73.

11. Kitaoka HB, Alexander IJ, Adelaar RS, Nunley JA, Myerson MS, Sanders M. Clinical rating systems for the ankle-hindfoot, midfoot, hallux and lesser toes. Foot Ankle Int. 1994;15(7): 349-53.

12. Thordarson DB, Krieger LE. Operative vs. nonoperative treatment of intra-articular fractures of the calcaneus: a prospective randomized trial. Foot Ankle Int. 1996;17(1):2-9.

13. Talarico LM, Vito GR, Zyryanov SY. Management of displaced intraarticular calcaneal fractures by using external ring fixation, minimally invasive open reduction, and early weightbearing. J Foot Ankle Surg. 2004;43(1):43-50.

14. Grala P, Machynska-Bucko Z, Kierzynka G. Surgical treatment of articular calcaneal fractures. Ortop Traumatol Rehabil. 2007; 9(1):89-97. English, Polish.

15. Schepers T, Schipper IB, Vogels LM, Giani AZ, Mulder PG, Heetveld MJ, Patka P. Percutaneous treatment of displaced intra-articular calcaneal fractures. J Orthop Sci. 2007;12(1): 22-7.

16. Wu Y, Yang MH, Wang JH, Wang MY, Sun ZW. [Open reduction and internal fixation of displaced intra-articular fractures of the calcaneus]. Zhonghua Wai Ke Za Zhi. 2005; 43(12):788-91. Chinese.

17. Herscovici D Jr, Widmaier J, Scaduto JM, Sanders RW, Walling A. Operative treatment of calcaneal fractures in elderly patients. J Bone Joint Surg Am. 2005;87(6):1260-4.

18. Aldridge JM 3rd, Easley M, Nunley JA. Open calcaneal fractures: results of operative treatment. J Orthop Trauma. 2004;18(1):7-11.

19. Moraes Filho DC, Provenzano E, Mattos JR, Batista LC, Galbiatti JA, Ferreira JCD, et al. Avaliação preliminar do tratamento cirúrgico de fraturas intra-articulares do calcâneo. Rev Bras Ortop. 1998;33(7):511-8.

20. Koberle G, Oliveira AC, Sandoval PS. Fraturas intra-articulares do calcâneo. Rev Bras Ortop. 1996;31(6):477-80.

21. Santin RAL, Fonseca Filho FF, Mercadante MT, Ferreira RC, Roncatto CE, Manzotti JP, et al. Tratamento operatório das fraturas articulares do calcâneo com placa "duplo H". Rev Bras Ortop. 1995;30(6):377-84

22. Murachovsky J, Martinelli MO, Ferreira RC, Fonseca Filho F. Fratura articular do calcâneo: resultado clínico-funcional do tratamento cirúrgico Rev Bras Ortop. 2000;35(8):314-24.

\footnotetext{
Declaração de inexistência de conflitos de interesse: Declaramos a inexistência de conflitos de interesse.
} 